\title{
Electrocardiogram Derived Respiration for Tracking Changes in Tidal Volume from a Wearable Armband*
}

\author{
Jesús Lázaro, Member, IEEE, Natasa Reljin, Member, IEEE, Raquel Bailón, Eduardo Gil, \\ Yeonsik Noh, Pablo Laguna Fellow, IEEE, Ki H. Chon, Senior Member, IEEE
}

\begin{abstract}
A pilot study on tracking changes in tidal volume (TV) using ECG signals acquired by a wearable armband is presented. The wearable armband provides three ECG channels by using three pairs of dry electrodes, resulting in a device that is convenient for long-term daily monitoring. An additional ECG channel was derived by computing the first principal component of the three original channels (by means of principal component analysis). Armband and spirometer signals were simultaneously recorded from five healthy subjects who were instructed to breathe with varying TV. Three electrocardiogram derived respiration (EDR) methods based on QRS complex morphology were studied: the QRS slopes range (SR), the $R$-wave angle $(\Phi)$, and the $R$-S amplitude (RS). The peak-to-peak amplitudes of these EDR signals were estimated as surrogates for TV, and their correlations with the reference TV (estimated from the spirometer signal) were computed. In addition, a multiple linear regression model was calculated for each subject, using the peak-to-peak amplitudes from the three EDR methods from the four ECG channels. Obtained correlations between TV and EDR peak-to-peak amplitude ranged from 0.0448 up to 0.8491 . For every subject, a moderate correlation $(>0.5)$ was obtained for at least one EDR method. Furthermore, the correlations obtained for the subject-specific multiple linear regression model ranged from 0.8234 up to 0.9154 , and the goodness of fit was $0.73 \pm 0.07$ (median \pm standard deviation). These results suggest that the peak-to-peak amplitudes of the EDR methods are linearly related to the TV. opening the possibility of estimating TV directly from an armband ECG device.
\end{abstract}

Clinical Relevance- This opens the door to possible continuous monitoring of TV from the armband by using EDR.

*This project has received funding from the European Union's Framework Programme for Research and Innovation Horizon 2020 (20142020) under the Marie Skłodowska-Curie Grant Agreement No. 745755. This work was also supported by Government of Aragón and European Social Fund (EU) through BSICoS group (T96), and by CIBER in Bioengineering, Biomaterials \& Nanomedicine (CIBER-BBN) through Instituto de Salud Carlos III. The computation was performed by the ICTS NANBIOSIS, specifically by the High Performance Computing Unit of CIBER-BBN at University of Zaragoza. This work was also supported by NSF SBIR Phase I (\#1746589) and R43 HL135961. Ki Chon is a cofounder of the Mobile Sense Technologies and the result presented in this work may benefit the company.

J. Lázaro (corresponding author), R. Bailón, E. Gil and P. Laguna are with the Biomedical Signal Interpretation and Computational Simulation (BSICoS) group at the Aragón Institute of Engineering Research (I3A), IIS Aragón, University of Zaragoza, Spain, and CIBER de Bioingeniería, Biomateriales y Nanomedicina (CIBER-BBN), Spain. (e-mail: jlazarop@unizar.es)

J. Lázaro, N. Reljin, and K. H. Chon are with the Biomedical Engineering Department, University of Connecticut, Storrs, CT 06269, USA.

Y. Noh is with the College of Nursing and with the Department of Electrical and Computer Engineering of the University of Massachussetts, Amherst, MA 01003, USA.

\section{INTRODUCTION}

Long-term continuous cardiorespiratory monitoring can be useful in several scenarios, including sleep studies, prediction of epileptic seizures, stress assessment, and monitoring of chronic respiratory patients [1]. Respiration is usually monitored by techniques that require cumbersome devices albeit recent advances in sensor technologies have made it possible for daily monitoring. However, they require separate respiration sensor and its data acquisition circuit. Thus, indirect assessment of respiratory information from other biomedical signals, such as electrocardiogram (ECG), has been extensively investigated [2]. The great majority of these studies are focused on respiratory rate, which is a sensitive clinical parameter in a multitude of pulmonary diseases [3]. However, tidal volume (TV) (the volume of air inhaled or exhaled during the respiratory cycle) is also a clinically relevant index, which is especially useful for monitoring respiratory issues such as the Cheney-Stokes respiration and sleep apnea [4]. The ideal respiratory monitor would provide continuous information on respiratory rate, $\mathrm{TV}$, and gas exchange in a non-obtrusive fashion [5].

There are scant studies on deriving TV from the ECG signal. Some authors reported proportionality between TV and an ECG derived respiration (EDR) signal based on R-S amplitude [6]. TV was estimated from 10 mechanicallyventilated Yorkshire swine using an EDR signal based on root-mean-square amplitude in [7]. In [4], TV was estimated from EDR signals based on both heart rate and beat morphology features during a treadmill test. However, the strong relationship between TV and heart rate during exercise limits the interpretation of the results, as the method could be mainly tracking changes in heart rates and not TV.

In this paper, a pilot study on how the changes in TV can be tracked by an ECG-based wearable device is presented. The wearable device is an armband recently developed in our lab at the University of Connecticut. This armband is designed to be worn on the left upper-arm, and it provides three ECG channels using three pairs of dry electrodes. The coverage (percentage of time providing usable data) of this device is approximately 50\% during the non-bed time, and 95\% during the bed time [8]. Furthermore, a previous study revealed that the respiratory-related modulations can be observed in the ECG signals acquired by the armband [1] Further details are provided about the armband in [8].

\section{MATERIALS AND METHODS}

\section{A. Signal acquisition}

The armband and spirometer signals were simultaneously recorded from five healthy volunteers during a lab-controlled 

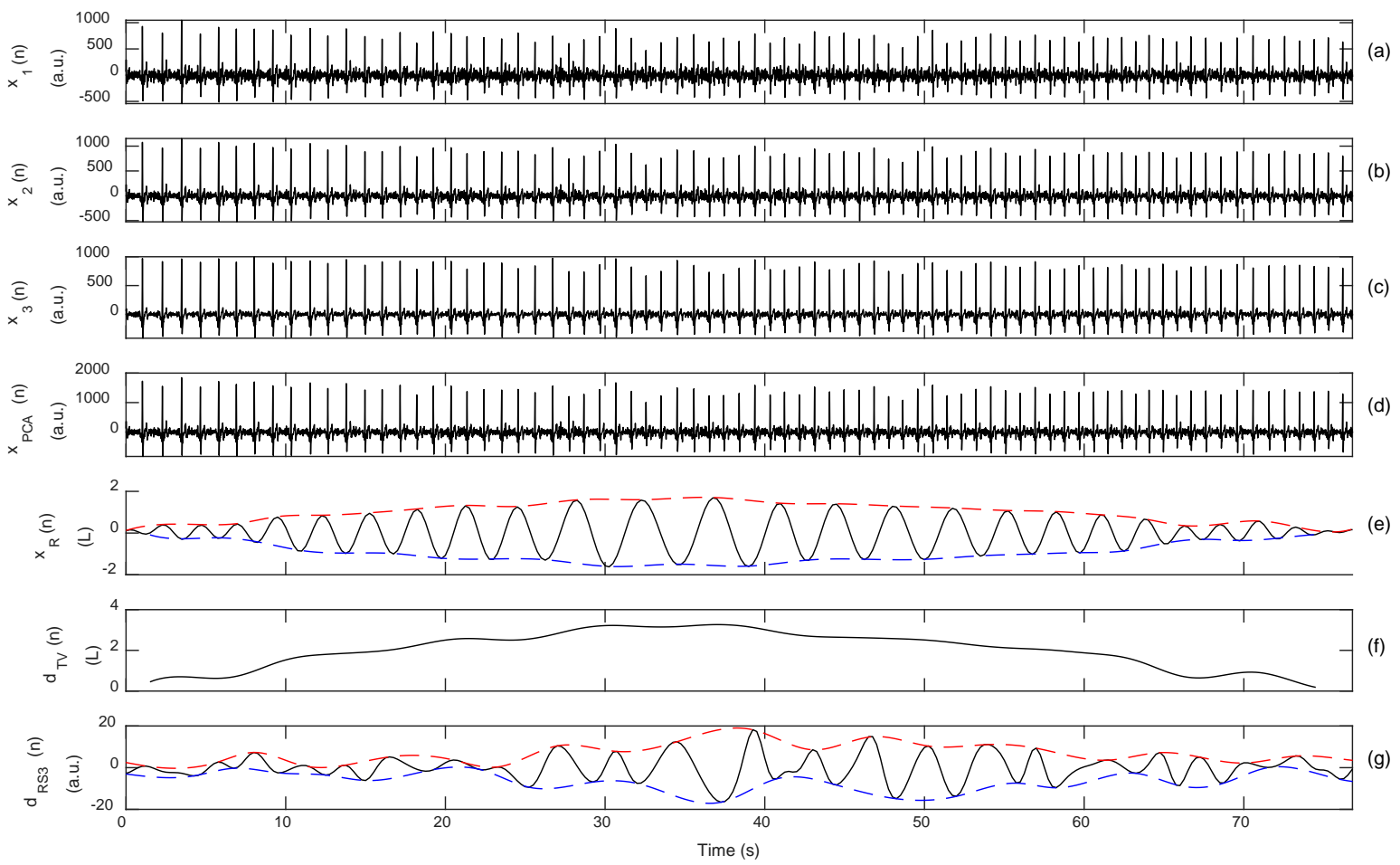

Figure 1. Example of acquired signals: (a), (b), and (c) are the three preprocessed armband-ECG channels $\mathrm{x}_{1}(\mathrm{n}), \mathrm{x}_{2}(\mathrm{n})$, and $\mathrm{x}_{3}(\mathrm{n})$, respectively. (d) shows the synthetized ECG channel (by means of PCA) $x_{P C A}(n)$. (e) shows the spirometry signal $x_{R}(n)$ and its estimated envelopes (in dashed red and blue lines). (f) shows the tidal volume estimated from $\mathrm{x}_{\mathrm{R}}(\mathrm{n})$ by subtracting its estimated envelopes. (g) shows the EDR signal whose peak-to-peak amplitude provided the highest correlation with TV in this subject: RS from $\mathrm{x}_{3}(\mathrm{n})$.

experiment which included an exercise consisting of breathing with different TV. Subjects were requested to breathe through the spirometer while looking at its output signal on a computer screen in order to have a visual feedback. The subjects were asked to begin breathe with a small TV while maintaining the same volume for three consecutive breathing cycles. The subjects were subsequently asked to incrementally increase their $\mathrm{TV}$ also while maintaining this depth for three consecutive cycles, and then to their maximum TV again for three consecutive cycles. The subjects were then asked to decrement their TV in a similar step wise fashion. An example of TV obtained during the above described breathing procedures can be observed in the panel (e) of Fig. 1.

\section{B. Signal preprocessing}

Armband-ECG signals were preprocessed as in [8]. This includes a bandpass filter with $3 \mathrm{~Hz}$ and $25 \mathrm{~Hz}$ as the cut-off frequencies. In addition, we estimated a new ECG channel from the three channels of the armband, by taking the first principal component from the spatial principal component analysis (PCA).

$\mathrm{R}$ peaks were automatically detected by a technique based on variable-frequency-complex-demodulation [9]. Then, the $\mathrm{Q}$ and $\mathrm{S}$ peaks were detected by an automatic delineator based on the discrete wavelet transform [10].

\section{Electrocardiogram derived respiration methods}

Three EDR techniques based on QRS morphology were applied to each one of the ECG channels. Thus, a total of 12 EDR signals were obtained per subject. These three EDR techniques are:

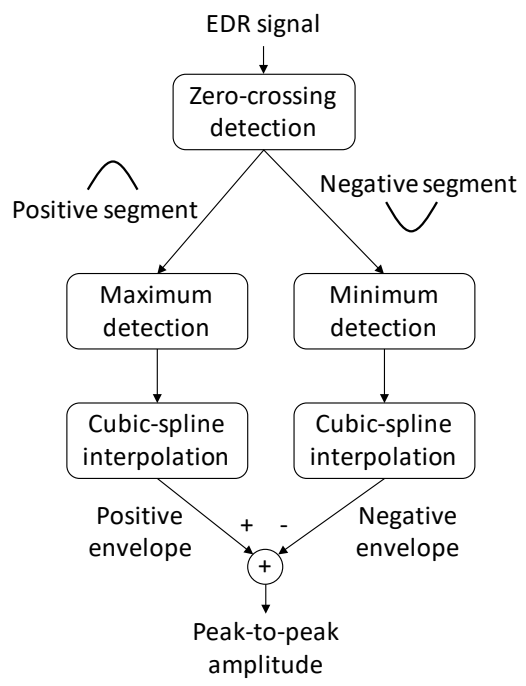

Figure 2. Flow diagram of the algorithm for estimating the peak-to-peak amplitude of an EDR signal. The sampling rate used was $4 \mathrm{~Hz}$.

$Q R S$ slope range (SR): It exploits the respiration-related variations of the QRS slopes. First, the QRS slopes are estimated from the first derivative of each QRS complex. Subsequently, the SR is defined as the maximum slope minus the minimum slope [11]. These SR series are known to be oscillating synchronously with respiration.

$R$-wave angle ( $\Phi)$ : Similar to the SR, this EDR technique exploits the respiration-related modulation of the QRS slopes. In this case, the R-wave angle is estimated as the smallest angle formed by two lines having an slope equal to the estimated QRS slopes [1]. 


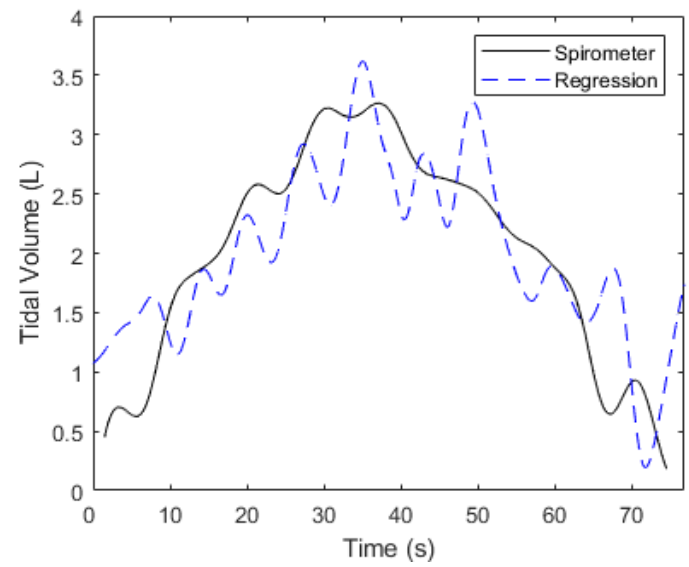

Figure 3. Example of tidal volume estimated by the multiple linear regression model (dashed blue line) vs. the tidal volume estimated from the spirometer signal (solid black line). In this case, obtained correlation was 0.8371 , and goodness of fit was 0.70 .

$R$-S amplitude (RS): Similarly to the two EDR methods described above, this EDR technique exploits the respirationrelated modulation of the QRS morphology. In this case, the amplitude of each QRS complex is estimated as the amplitude of the $\mathrm{R}$ peak with respect to the amplitude of the $\mathrm{S}$ peak. This parameter is also known to be oscillating synchronously with respiration [6].

\section{Tidal volume tracking}

The hypothesis underlying this work is that the peak-topeak amplitude of the EDR signals is related to the depth of breathing and thus, to the TV. An evenly sampled version of the EDR series (which are measured beat-to-beat) was obtained using the cubic-splines interpolation with a sampling rate of $4 \mathrm{~Hz}$. Afterwards, a band-pass filter with the cut-off frequencies of $0.075 \mathrm{~Hz}$ and $1 \mathrm{~Hz}$ was applied. This processing remains the same from those used in [1] for estimating respiratory rate from the armband-ECG signals.

The peak-to-peak amplitude was then estimated from each one of the evenly-sampled EDR signals, by subtracting its positive and negative envelopes. These envelopes were estimated from the local maxima (positive envelope) and local minima (negative envelope) between zero-crossings. A flow diagram of the algorithm is shown in Fig. 2.

The spirometer signal was resampled to the same sampling rate of the EDR signals $(4 \mathrm{~Hz})$. Furthermore, the same band-pass filter $(0.075-1 \mathrm{~Hz})$ was applied. The reference TV was estimated as the peak-to-peak amplitude of the resulting band-pass-filtered spirometer signal and used as the gold standard for evaluation of different EDR methods. An example of the positive and negative envelopes obtained from a spirometer signal is shown in Fig. 1e, and the resulting reference TV is shown in Fig. 1f.

\section{E. Performance measures}

In order to assess the capability of the EDR methods to track the changes in TV, the correlation between the reference TV (estimated from the spirometer signal) and the EDR's peak-to-peak amplitude was computed.

In addition, a subject-specific multiple linear regression model using the peak-to-peak amplitudes of all EDR signals derived from all ECG channels was used to predict TV. An example of the TV estimated by the subject-specific multiple linear regression model can be observed in Fig. 3.

\section{RESUlTS}

Table I shows the obtained correlations between the TV and the peak-to-peak amplitude of the different EDR methods, for every subject and ECG channel. In addition, the correlations obtained from the subject-specific multiple linear regression model are also shown in Table I. The goodness of fit $\left(R^{2}\right)$ for the multiple linear regression were $0.70,0.75,0.84,0.68$, and 0.69 , in case of subjects $1,2,3,4$, and 5 , respectively.

TABLE I. CORRELATIONS BETWEEN TV AND THE PEAK-TO-PEAK AMPLITUDES FOR ALL SUBJECTS.THE ROWS REPRESENT EACH OF THE THREE EDR METHODS FOR EACH OF THE FOUR CHANNELS. IN ADDITION, THE CORRELATIONS OBTAINED FROM THE ADJUSTMENT OF THE MULTIPLE LINEAR REGRESSION IS ALSO SHOWN IN THE BOTTOM ROW.

\begin{tabular}{|c|ccccc|}
\cline { 2 - 6 } \multicolumn{1}{c|}{} & \multicolumn{5}{c|}{ Subjects } \\
\cline { 2 - 6 } \multicolumn{1}{c|}{} & 1 & 2 & 3 & 4 & 5 \\
\hline $\mathrm{SR}_{1}$ & 0.5513 & 0.5320 & 0.6882 & 0.5556 & 0.3224 \\
$\Phi_{1}$ & 0.4534 & $\mathbf{0 . 7 4 7 7}$ & $\mathbf{0 . 7 7 1 8}$ & 0.5933 & 0.4836 \\
$\mathrm{RS}_{1}$ & 0.2805 & 0.6780 & $\mathbf{0 . 8 4 3 8}$ & 0.2038 & 0.0448 \\
\hline $\mathrm{SR}_{2}$ & 0.6178 & 0.6876 & 0.6644 & 0.0091 & 0.1550 \\
$\Phi_{2}$ & 0.5398 & 0.6708 & $\mathbf{0 . 8 6 3 3}$ & 0.1815 & 0.6086 \\
$\mathrm{RS}_{2}$ & 0.5035 & 0.5938 & $\mathbf{0 . 8 2 9 1}$ & 0.1880 & 0.4064 \\
\hline $\mathrm{SR}_{3}$ & 0.4669 & $\mathbf{0 . 7 0 4 6}$ & 0.6538 & 0.3361 & 0.2103 \\
$\Phi_{3}$ & 0.6368 & 0.4442 & 0.6302 & 0.2202 & 0.3832 \\
$\mathrm{RS}_{3}$ & $\mathbf{0 . 8 3 4 4}$ & 0.4069 & $\mathbf{0 . 7 2 7 4}$ & 0.3194 & 0.2087 \\
\hline $\mathrm{SR}_{\text {PCA }}$ & 0.6319 & 0.6571 & 0.6855 & 0.1255 & 0.3195 \\
$\Phi_{\text {PCA }}$ & 0.5461 & 0.4369 & $\mathbf{0 . 8 0 4 9}$ & 0.2994 & $\mathbf{0 . 7 4 1 5}$ \\
$\mathrm{RS}_{\text {PCA }}$ & 0.4942 & $\mathbf{0 . 7 8 5 3}$ & $\mathbf{0 . 8 4 9 1}$ & 0.2658 & 0.5164 \\
\hline Regression & $\mathbf{0 . 8 3 7 1}$ & $\mathbf{0 . 8 6 8 1}$ & $\mathbf{0 . 9 1 5 4}$ & $\mathbf{0 . 8 2 3 4}$ & $\mathbf{0 . 8 3 1 7}$ \\
\hline
\end{tabular}

\section{DISCUSION}

A pilot study on tracking changes in TV from ECG signals acquired by a wearable armband has been presented. The armband provides three ECG channels by using three pairs of dry electrodes, resulting in a device that is very convenient for long-term daily monitoring. An additional ECG channel created by estimating the first principal component of the three original channels (by means of PCA). This armband can provide approximately $50 \%$ of usable data during the non-bed time, and $95 \%$ of usable data during the bed time [8]. Armband and spirometer signals were simultaneously recorded from five healthy subjects in which they were requested to breathe with different TV.

Three EDR methods based on QRS complex morphology were studied: SR, $\Phi$, and RS. The features on which SR is based (QRS slopes), as well as $\Phi$, were previously used for estimating respiratory rate from the armband-ECG signals, obtaining a relative error that was lower than 5\% [1]. In addition, the RS method was also studied because its simplicity makes it very interesting for wearable devices. The hypothesis underlying this work is that the peak-to-peak amplitude of the EDR signals is related to the depth of breathing and thus, to the TV.

The spirometer signal was used as the reference TV for evaluating EDR methods. The peak-to-peak amplitude of the EDR signals was estimated, and their correlation with the reference TV was computed. Obtained correlations ranged 
from 0.0448 up to 0.8491 . The SR-based EDR method obtained moderate correlation $(>0.5)$ in 12 (of the 20 possible) cases. The $\Phi$-based EDR method obtained moderate correlations in 12 cases, achieving strong correlation $(>0.7)$ in 5 of those cases. The RS-based EDR method obtained moderate correlations in 10 cases, achieving strong correlation in 6 cases. For every subject, moderate correlation was obtained for at least one of the EDR methods. These results suggest that the peak-to-peak amplitude of the studied EDR methods is linearly related to the TV. However, no EDR method obtained consistently high correlation for all the subjects. Similarly, no ECG channel obtained consistently high correlations. These results suggest that a final application may require a subjectspecific setup.

A multiple linear regression model was adjusted for each subject, using the peak-to-peak amplitude from the three EDR methods from the four ECG channels. Obtained correlations were from the lowest of 0.8234 to as high as 0.9154 , demonstrating the linear relationship of the TV with the peak-to-peak amplitudes. These results suggest that the wearable armband can track the changes in TV, although two limitations of this study should be noted. One limitation is that the low number of subjects used for this study. Another limitation is that the multiple linear regression model was not tested with independent data, so the results may be biased due to a possible overfitting. Further studies are needed, including more subjects and more diverse breathing experiments per subject to determine the true efficacy of the proposed approach in approximating TV from EDR-derived parameters from ECG signals.

Obtained $R^{2}$ were slightly lower than those reported in [7] for mechanically-ventilated Yorkshire swine $(0.73 \pm 0.07$ vs. $0.77 \pm 0.11$ in median \pm standard deviation). It should be noted that the ECG signals analyzed in this paper are recorded by three pairs of electrodes over the left upper-arm that are integrated into a wearable armband. This represents a more challenging scenario than the conventional ECG setup, which is based on wet (hydrogel) electrodes over the chest, although the armband is a much more convenient device alternative for long-term daily monitoring.

It should be noted that, the algorithm to estimate the amplitude of EDR signals was designed for tracking fast changes (as envelopes are estimated peak-to-peak), while an estimation of the trend (slower variations, e.g., minute-tominute) would be enough for some applications, such as daily monitoring of patients with chronic respiratory problems. This fact is important because the peak-to-peak amplitude of the EDR signals were observed to be noisy, while tracking the slower changes was better than the faster ones (see Fig. 3 as an example). These observations suggest that the armband would be more appropriate for this kind of applications. On the contrary, other applications would require tracking fast variations, such as sleep apnea detection, where periods of 10 seconds (or longer) with cessation of breathing have to be detected. The temporal resolution of the EDR amplitude estimation should be adopted for each application.

\section{CONCLUSION}

The results suggest that the peak-to-peak amplitude of the EDR methods derived from the armband ECG is linearly related to the TV. This preliminary study suggests a good potential for tracking changes in TV directly from the armband device, which is a more conducive approach for long-term monitoring when compared to a Holter monitor. Further studies are needed in order to validate the present findings. These studies should include a higher number of subjects, and an evaluation of the multiple linear regression model with different data rather than the actual source data.

\section{REFERENCES}

[1] J. Lázaro, R. Bailón, E. Gil, Y. Noh, P. Laguna, and K. H. Chon, "Pilot Study on Electrocardiogram Derived Respiratory Rate Using a Wearable Armband," in 2018 Computing in Cardiology Conference (CinC), 2018, vol. 45, pp. 1-4, doi: 10.22489/CinC.2018.054.

[2] P. H. Charlton, T. Bonnici, L. Tarassenko, D. A. Clifton, R. Beale, and P. J. Watkinson, "An assessment of algorithms to estimate respiratory rate from the electrocardiogram and photoplethysmogram,” Physiol. Meas., vol. 37, no. 4, pp. 610-626, Apr. 2016, doi: 10.1088/0967-3334/37/4/610.

[3] B. Krieger, D. Feinerman, A. Zaron, and F. Bizousky, "Continuous noninvasive monitoring of respiratory rate in critically ill patients," Chest, vol. 90, no. 5, pp. 632-634, Nov. 1986, doi: 10.1378/chest.90.5.632.

[4] J. Milagro et al., "Electrocardiogram-Derived Tidal Volume During Treadmill Stress Test," IEEE Trans. Biomed. Eng., vol. 67, no. 1, pp. 193-202, Jan. 2020, doi: 10.1109/TBME.2019.2911351.

[5] M. Folke, L. Cernerud, M. Ekström, and B. Hök, "Critical review of non-invasive respiratory monitoring in medical care," Med. Biol. Eng. Comput., vol. 41, no. 4, pp. 377-383, Jul. 2003, doi: $10.1007 / \mathrm{bf} 02348078$.

[6] G. B. Moody, R. G. Mark, A. Zoccola, and S. Mantero, "Derivation of Respiratory Signals from Multi-lead ECGs," presented at the Computers in Cardiology, 2008.

[7] O. Sayadi, E. H. Weiss, F. M. Merchant, D. Puppala, and A. A. Armoundas, "An optimized method for estimating the tidal volume from intracardiac or body surface electrocardiographic signals: implications for estimating minute ventilation,” Am. J. Physiol. Heart Circ. Physiol., vol. 307, no. 3, pp. H426-436, Aug. 2014, doi: 10.1152/ajpheart.00038.2014.

[8] J. Lazaro, N. Reljin, Y. Noh, P. Laguna, and K. H. Chon, "Feasibility of Long-Term Daily Life Electrocardiogram Monitoring Based on a Wearable Armband Device," Conf. Proc. Annu. Int. Conf. IEEE Eng. Med. Biol. Soc. IEEE Eng. Med. Biol. Soc. Annu. Conf., vol. 2019, pp. 4314-4317, Jul. 2019, doi: 10.1109/EMBC.2019.8857219.

[9] S. K. Bashar, A. J. Walkey, D. D. McManus, and K. H. Chon, "VERB: VFCDM-Based Electrocardiogram Reconstruction and Beat Detection Algorithm,” IEEE Access Pract. Innov. Open Solut., vol. 7, pp. 13856-13866, 2019, doi: 10.1109/ACCESS.2019.2894092.

[10] J. P. Martínez, R. Almeida, S. Olmos, A. P. Rocha, and P. Laguna, "A wavelet-based ECG delineator: evaluation on standard databases," IEEE Trans. Biomed. Eng., vol. 51, no. 4, pp. 570-581, Apr. 2004, doi: 10.1109/TBME.2003.821031.

[11] S. Kontaxis et al., "ECG-derived Respiratory Rate in Atrial Fibrillation,” IEEE Trans. Biomed. Eng., Jun. 2019, doi: 10.1109/TBME.2019.2923587. 